\title{
Research Article Kinematic Selection Criteria in the Search for Resonances
}

\author{
B. B. Levchenko \\ Skobeltsyn Institute of Nuclear Physics, Lomonosov Moscow State University, Moscow 119234, Russia
}

Correspondence should be addressed to B. B. Levchenko, levtchen@mail.desy.de

Received 30 April 2012; Accepted 5 June 2012

Academic Editor: C. Q. Geng

Copyright $@ 2012$ B. B. Levchenko. This is an open access article distributed under the Creative Commons Attribution License, which permits unrestricted use, distribution, and reproduction in any medium, provided the original work is properly cited.

In this paper, we discuss some general features of two-body decay kinematics and derive a new set of equations describing boundaries of the physical region in decays. These features via a graphical representation and implementation of selection criteria provide an efficient tool for the background suppression in experimental searches for conventional and exotic (pentaquark, etc.) resonance states.

\section{Introduction}

In the experimental search for a resonance production in particle collisions, momenta of secondary particles are combined to form the momentum of a resonance candidate. In high-energy reactions with high multiplicity of final states, this method of resonance reconstruction may cause a large combinatorial background which makes searches for new narrow states in invariant-mass distributions to be rather difficult. This was the case with the top-quark discovery, searches for new charmonium states or pentaquark searches. For reconstruction of the signal with a sufficient statistical significance, any additional physical information about the resonance and its decay properties (lifetime, the mass of secondary particles, etc.) helps considerably to reduce the background. For instance, the presence in an event a well-separated secondary vertex allows us to reconstruct $\Lambda, K_{s}^{0}$ and mesons from the $D$-meson family with a very low background. One such example is the study of meson resonances in the $K_{s}^{0} K_{s}^{0}$ final state in ep deep inelastic scattering at HERA [1]. Moreover, if masses of decay products are known and significantly differ from each other, this information also has to be used to reduce the background. This paper is a discussion of these issues.

Below only two-body decay modes are considered, and for each final state particle a set of kinematic equations describing boundaries of the physical region is given. Proposed selection criteria are based on the features of each physical region [2] and proved to be 
the efficient tool for experimental reconstruction both the conventional as well as exotic resonances $[3,4]$.

\section{Two-Particle Decay}

Let us consider a two-particle decay, $R \rightarrow \mathbf{a}+\mathbf{b}$, of a resonance $R$ with a mass $M_{R}$ and a momentum $\vec{P}_{R}$ in the laboratory frame. Masses of the decay products are denoted by $m_{\mathrm{a}}$ and $m_{\mathbf{b}}$. At the rest frame of $R$ the particles, $\mathbf{a}$ and $\mathbf{b}$ are flying in opposite directions with the momentum $[5,6]$ :

$$
P^{*}=\frac{1}{2 M_{R}}\left[\left(M_{R}^{2}-m_{\mathrm{a}}^{2}-m_{\mathrm{b}}^{2}\right)^{2}-4 m_{\mathrm{a}}^{2} m_{\mathrm{b}}^{2}\right]^{1 / 2} .
$$

In the laboratory frame, the absolute momenta $p_{\mathbf{a}}$ and $p_{\mathbf{b}}$ of the particles $\mathbf{a}$ and $\mathbf{b}$ depend on the relative orientation of the rest frame vectors $\vec{p}_{\mathbf{a}}^{*}$ and $\vec{p}_{\mathbf{b}}^{*}$ with respect to the boost vector. We shall consider only Lorentz boosts along the momentum $\vec{P}_{R}$ and denote by $\theta_{\mathbf{a}}^{*}$ the polar angle between $\vec{p}_{\mathbf{a}}^{*}$ and the direction given by $\vec{P}_{R}$. In that case, $\theta_{\mathrm{b}}^{*}=\pi-\theta_{\mathbf{a}}^{*}$.

The energy and momentum components in both frames are related via $[5,6]$ :

$$
\begin{gathered}
E_{\mathbf{a}(\mathbf{b})}=\gamma E_{\mathbf{a}(\mathbf{b})}^{*}-\vec{\eta} \cdot \vec{p}_{\mathbf{a}(\mathbf{b}))^{\prime}}^{*} \\
\vec{p}_{\mathbf{a}(\mathbf{b})}=\vec{p}_{\mathbf{a}(\mathbf{b})}^{*}+\vec{\eta}\left[\frac{\vec{\eta} \cdot \vec{p}_{\mathbf{a}(\mathbf{b})}^{*}}{\gamma+1}-E_{\mathbf{a}(\mathbf{b})}^{*}\right] .
\end{gathered}
$$

For a boost along $\vec{P}_{R}$, the boost parameters are

$$
\gamma=\frac{E_{R}}{M_{R}}, \quad \vec{\eta}=-\frac{\vec{P}_{R}}{M_{R}}
$$

and therefore

$$
p_{\mathbf{a}(\mathbf{b})}=\frac{1}{M_{R}} \sqrt{\left(E_{R} E_{\mathbf{a}(\mathbf{b})}^{*}+P_{R} P^{*} \cos \theta_{\mathbf{a}(\mathbf{b})}^{*}\right)^{2}-m_{\mathbf{a}(\mathbf{b})}^{2} M_{R}^{2}} .
$$

If in an experiment there is no possibility to determine a particle type corresponding to a given charged track, then only information about the particle momentum (2.5) is used. In the opposite case, when the particle type can be identified, for instance, by ionization and time of flight, one may incorporate this information and in addition use (2.2). Below, these cases are considered separately. 


\subsection{Particles without Identification}

The boundaries of the physical regions of the particle a on the $\left(P_{R}, p_{\mathbf{a}}\right)$ plane are easy to obtain with the use of (2.5). For a given $P_{R}, p_{\mathbf{a}}$ reaches the upper limit at $\theta_{\mathbf{a}}^{*}=0$ :

$$
p_{\mathbf{a}}^{+}=\frac{1}{M_{R}}\left(P_{R} E_{\mathbf{a}}^{*}+E_{R} P^{*}\right),
$$

when the lower limit at $\theta_{\mathbf{a}}^{*}=\pi$ :

$$
p_{\mathbf{a}}^{-}=\frac{1}{M_{R}}\left|P_{R} E_{\mathbf{a}}^{*}-E_{R} P^{*}\right|
$$

The equations similar to (2.6)-(2.7) are valid for the particle $\mathbf{b}$ too. At the resonance rest frame, (2.7) demonstrates an interesting feature of the momentum of the particle flying backward. With increasing $P_{R}$, the momentum $p_{\mathbf{a}}\left(p_{\mathbf{b}}\right)$ first decreases, at $P_{R}=M_{R} P^{*} / m_{\mathbf{a}(\mathbf{b})}$ it reaches the zero value and only at lager $P_{R}$ starts to increase. When plotted on the momentum $\left(P_{R}, p_{\mathrm{a}}\right)$ plane, (2.6)-(2.7) select a band-like physical region ( $m$-band). For secondary particles with equal masses, $m_{\mathbf{a}}=m_{\mathbf{b}}$, these $m$-bands are fully overlapping. However, if for instance, $m_{\mathbf{a}}>$ $m_{\mathbf{b}}$, the $m$-bands overlap only partially or even separate out at

$$
P_{R} \geq P_{R}^{(s)}=\frac{2 M_{R} P^{*}}{\sqrt{\left(E_{\mathbf{a}}^{*}-E_{\mathbf{b}}^{*}\right)^{2}-4\left(P^{*}\right)^{2}}} .
$$

The last equation follows from the condition $p_{\mathbf{a}}^{-} \geq p_{\mathbf{b}}^{+}$. In (2.8), the expression under the square root is positive only if

$$
P^{*}<\frac{m_{\mathbf{a}}^{2}-m_{\mathbf{b}}^{2}}{\sqrt{8\left(m_{\mathbf{a}}^{2}+m_{\mathbf{b}}^{2}\right)}} .
$$

Thus, for $m_{\mathbf{a}} \gg m_{\mathbf{b}}$ the physical regions of the particles $\mathbf{a}$ and $\mathbf{b}$ do not overlap if

$$
m_{\mathrm{a}} \geq \sqrt{8} P^{*}
$$

and $P_{R}>P_{R}^{(s)}$.

We shall now consider a few particular applications of (2.6)-(2.10). According to the PDG [7] mass values, only resonances with mass close to the threshold value $m_{\mathbf{a}}+m_{\mathbf{b}}$ satisfy the condition (2.10). Some of them are listed in Table 1 , where $P^{*}$ and $P_{R}^{(s)}$ values were calculated with use of (2.1) and (2.8), respectively.

Figures $1(\mathrm{a}), 1(\mathrm{~b})$, and $1(\mathrm{c})$ shows $m$-bands of decay products for some resonances listed in Table 1. For all of them always

$$
p_{\mathbf{a}}>p_{\mathbf{b}}
$$




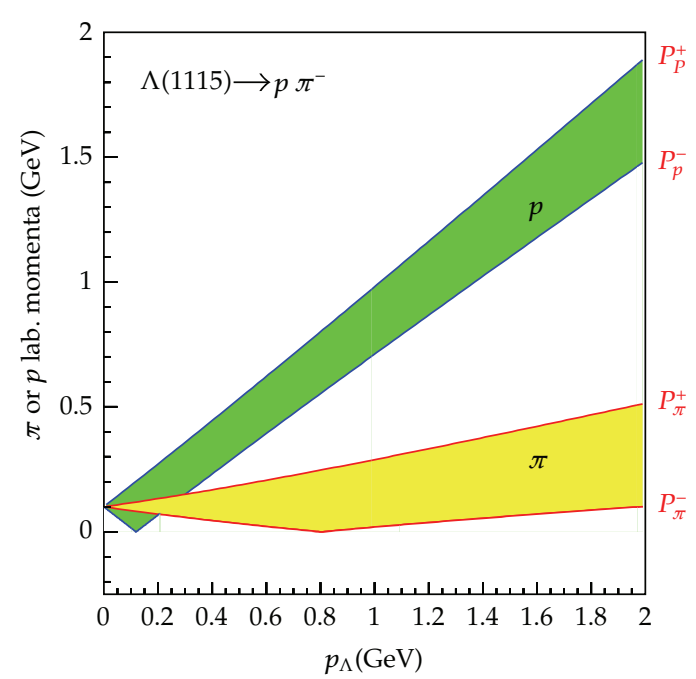

(a)

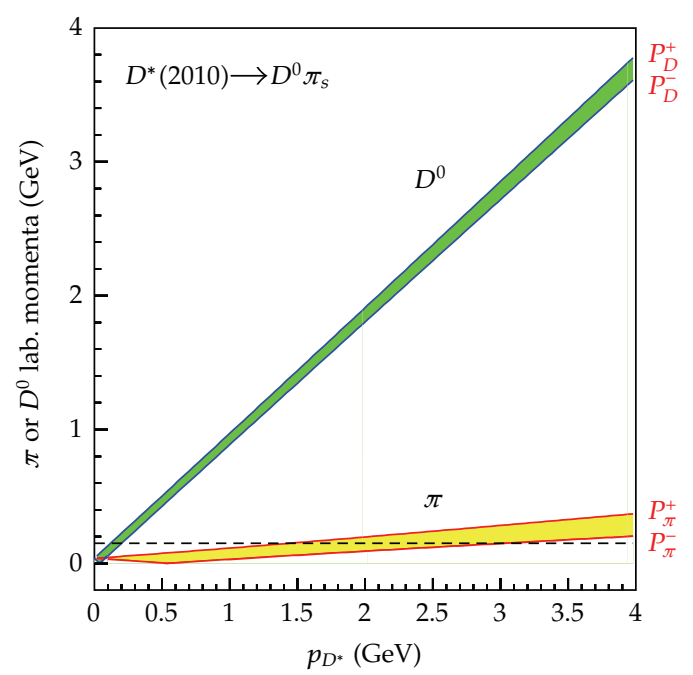

(c)

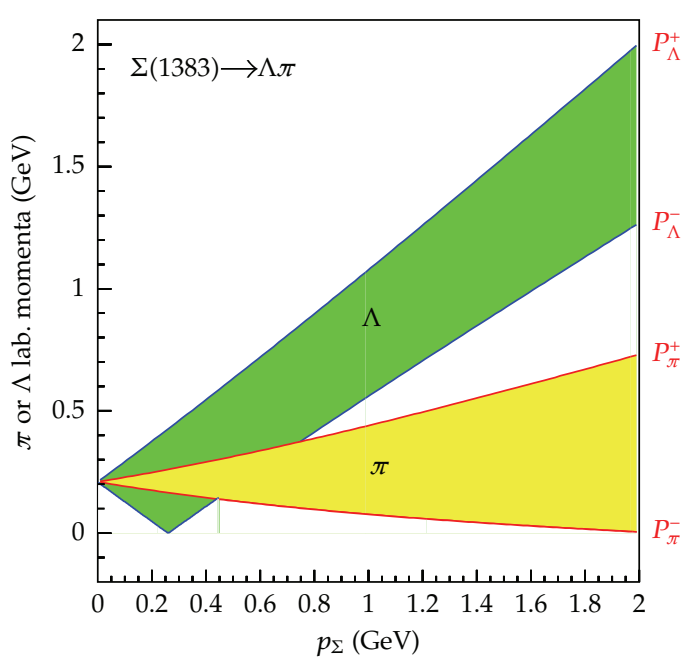

(b)

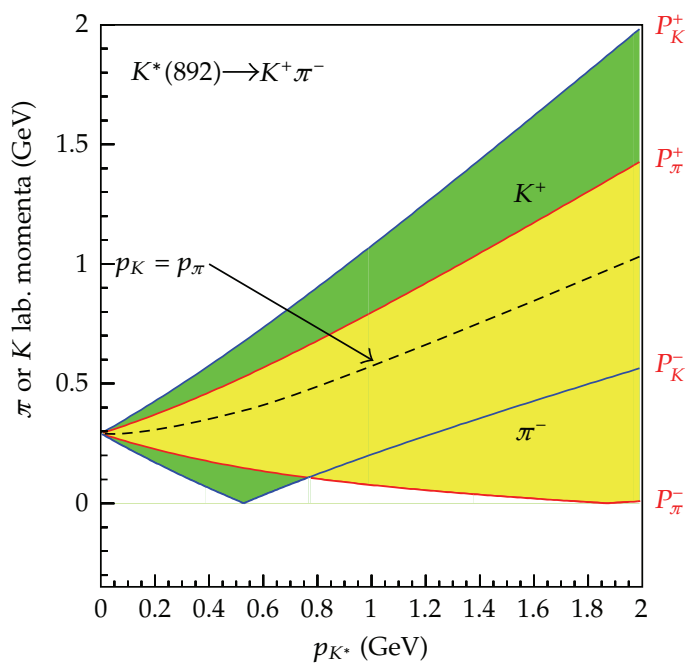

(d)

Figure 1: Phase space $m$-bands of the particles $\mathbf{a}$ and $\mathbf{b}$ in $R \rightarrow \mathbf{a}+\mathbf{b}$ decays as a function of the resonance momentum $P_{R}$. The dashed line on (c) corresponds to the momentum cut $p_{\pi}=0.15 \mathrm{GeV}$ (see Section 3). The dashed line on $(\mathrm{d})$ corresponds to (2.12).

at $P_{R}>P_{R}^{(s)}$, and $m_{\mathrm{a}}$ significantly larger $m_{\mathbf{b}}$. We will refer to the momentum condition (2.11) as a $m$-selector. Thus, for resonances of the type in Table 1, fulfillment of the condition (2.11) allows an assignment to the particle a of the mass $m_{\mathbf{a}}$, that is, we identify the particle a. The $m$-selector (2.11) is a powerful condition for the background suppression.

In the two-body decay modes, one meets with a higher incidence the situation shown in Figure 1(d). For these decays the conditions (2.9)-(2.10) are not fulfilled, and at any $P_{R}$ the phase space bands remain overlapping. Nevertheless, if $m_{\mathbf{a}}>m_{\mathbf{b}}$, one can demand fulfillment of (2.11) for the particle a in order to assign it the mass $m_{\mathbf{a}}$. In course of the resonance search, the condition (2.11) rejects not only a significant part of the background, but also some 
Table 1: The physical regions of the particles a and $\mathbf{b}$ do not overlap if $m_{a} \geq \sqrt{8} P^{*}$ and $P_{R}>P_{R}^{(s)}$. Here $M_{R}$ is the resonance mass, and $m_{a}$ is the mass of the heaviest product, $P^{*}$ is the rest frame momentum of the resonance $R$ decay products, and $P_{R}^{(s)}$ is the threshold momentum of the $m$-bands separation (8).

\begin{tabular}{lcccc}
\hline$R \rightarrow \mathbf{a}+\mathbf{b}$ & $M_{R}, \mathrm{GeV}$ & $m_{\mathbf{a}}, \mathrm{GeV}$ & $\sqrt{8} P^{*}, \mathrm{GeV}$ & $P_{R}^{(s)}, \mathrm{GeV}$ \\
\hline$\Lambda \rightarrow p+\pi^{-}$ & 1.115 & 0.938 & 0.284 & 0.301 \\
$\Delta \rightarrow N+\pi$ & 1.232 & 0.939 & 0.643 & 1.052 \\
$\Sigma \rightarrow N+\pi$ & 1.193 & 0.939 & 0.528 & 0.714 \\
$\Sigma \rightarrow \Lambda+\pi$ & 1.385 & 1.115 & 0.596 & 0.752 \\
$\Sigma \rightarrow N+K$ & 1.480 & 0.939 & 0.494 & 2.066 \\
$D^{0} \rightarrow a_{1}(1260)^{+}+K^{-}$ & 1.865 & 1.230 & 0.924 & 6.457 \\
$D^{* \pm} \rightarrow D^{0}+\pi^{ \pm}$ & 2.010 & 1.865 & 0.107 & 0.088 \\
$D_{s 1}^{+} \rightarrow D^{*}(2010)^{+}+K^{0}$ & 2.535 & 2.010 & 0.424 & 0.519 \\
$\ldots$ & $\ldots$ & $\cdots$ & $\ldots$ & $\ldots$ \\
\hline
\end{tabular}

fraction of the signal combinations. To estimate the efficiency of the $m$-selector we proceed in the following way.

On $\left(P_{R}, p_{\mathrm{a}}\right)$ plane, the condition (2.11) is valid up to the line defined by the equation:

$$
p_{\mathbf{a}}\left(\cos \theta_{\mathbf{a}}^{*}, P_{R}\right)=p_{\mathbf{b}}\left(\cos \theta_{\mathbf{b}}^{*}, P_{R}\right)
$$

For $K^{*} \rightarrow K \pi$ decays the last equality is shown in Figure $1(\mathrm{~d})$ by the dashed line. The true $K^{*}$ is a combination of a kaon from the region above the line (2.12) with a pion below it and vice versa. From (2.12) and (2.5), we find the variation of $\theta_{\mathbf{a}}^{*}=\pi-\theta_{\mathbf{b}}^{*}$ along the line (2.12):

$$
\cos \widehat{\theta}_{\mathbf{a}}^{*}=-\frac{P_{R}}{E_{R}} \cdot \frac{\left(E_{\mathbf{a}}^{*}-E_{\mathbf{b}}^{*}\right)}{2 P^{*}}
$$

At the rest frame of $R,(2.11)$ is equivalent to the exclusion of the region $\theta_{\mathbf{a}}^{*}>\widehat{\theta}_{\mathbf{a}}^{*}$. For unpolarized particles the distribution of $\cos \theta_{\mathbf{a}}^{*}$ is uniform, and we define the efficiency of the $m$-selector (2.11) as

$$
\mathrm{Eff}=\frac{1-\cos \widehat{\theta}_{\mathbf{a}}^{*}}{2} \cdot 100 \%
$$

With the definition (2.14), we get Eff $=100 \%$, when the conditions (2.9)-(2.10) are fulfilled, and Eff $=50 \%$ for decays with $m_{\mathbf{a}}=m_{\mathbf{b}}$ (for real data Eff $<50 \%$, see discussion in Section 3). As another example, in Figure 4 by the dashed line is shown the evolution of Eff with $P_{R}$ in $K^{*} \rightarrow K \pi$ decays. Eff grows because with the increase of $P_{R}$ the overlap of $m$-bands decreases. 
In the limit large $P_{R}$, the expression for the efficiency can be simplified:

$$
\begin{aligned}
\text { Eff } & =\frac{1}{2}\left(1+\frac{m_{\mathbf{a}}^{2}-m_{\mathbf{b}}^{2}}{\sqrt{\left[M_{R}^{2}-\left(m_{\mathbf{a}}-m_{\mathbf{b}}\right)^{2}\right]\left[M_{R}^{2}-\left(m_{\mathbf{a}}+m_{\mathbf{b}}\right)^{2}\right]}}\right) \cdot 100 \% \\
& \simeq \frac{1}{2} \cdot \frac{M_{R}^{2}}{M_{R}^{2}-m_{\mathbf{a}}^{2}} \cdot 100 \% .
\end{aligned}
$$

The last equation is a good approximation only if $m_{\mathbf{a}} \gg m_{\mathbf{b}}$. Equations (2.15)-(2.16) confirm the result we have already seen in Figure 4(a), where Eff is independent of $P_{R}$ at large $P_{R}$. On the other hand, for fixed values of $m_{\mathbf{a}}$ and $m_{\mathbf{b}}$, Eff decreases with increasing $M_{R}$, the mass of the resonance candidate. Thus, the higher the invariant mass of a two-particle combination, the more strongly (2.11) suppresses that part of the mass spectrum.

\subsection{Identified Particles}

Accounting for the particle masses transforms (2.6)-(2.7) into

$$
\begin{aligned}
& E_{\mathbf{a}}^{+}=\frac{1}{M_{R}}\left(E_{R} E_{\mathbf{a}}^{*}+P_{R} P^{*}\right), \\
& E_{\mathbf{a}}^{-}=\frac{1}{M_{R}}\left(E_{R} E_{\mathbf{a}}^{*}-P_{R} P^{*}\right)
\end{aligned}
$$

and at low $P_{R}$ leads to a "repulsion" between the phase space $E$-bands on the energy $\left(P_{R}, E_{\mathbf{a}}\right)$ plane (Figure 2). For all resonances with $m_{\mathbf{a}} \gg m_{\mathbf{b}}$ (see Table 1):

$$
E_{\mathbf{a}}>E_{\mathbf{b}}
$$

independent of $P_{R}$. This is not always true for the background combinations. Therefore, if applied, the condition (2.18) suppresses the background even more strongly than (2.11). We will refer to the energy condition (2.18) as an $E$-selector.

As in the previous section, if the masses of the secondary particles do not differ significantly, at $P_{R}$ greater than

$$
\widehat{P}_{R}=\frac{M_{R}\left(E_{\mathbf{a}}^{*}-E_{\mathbf{b}}^{*}\right)}{\sqrt{4\left(P^{*}\right)^{2}-\left(E_{\mathbf{a}}^{*}-E_{\mathbf{b}}^{*}\right)^{2}}}
$$

the E-bands start overlapping in the way shown in Figure 2(d). The loss of signal combinations by demanding (2.18) for resonance candidates we estimate again with (2.14). 


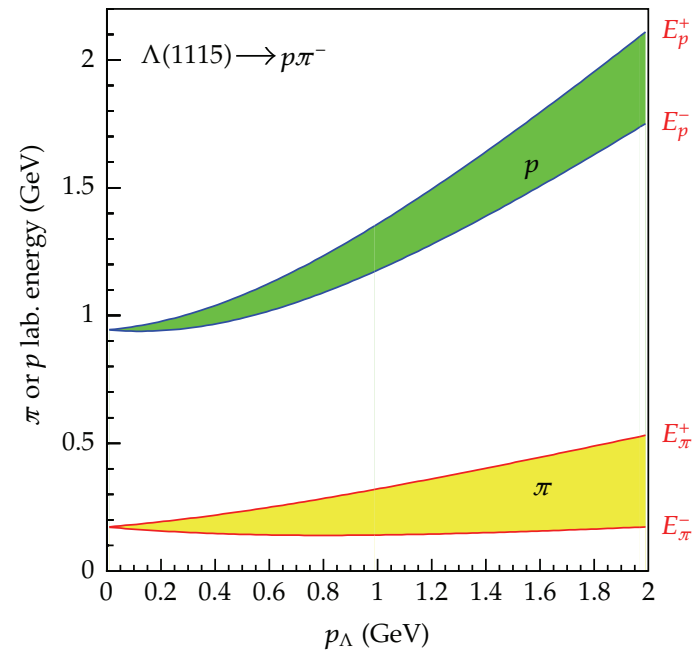

(a)

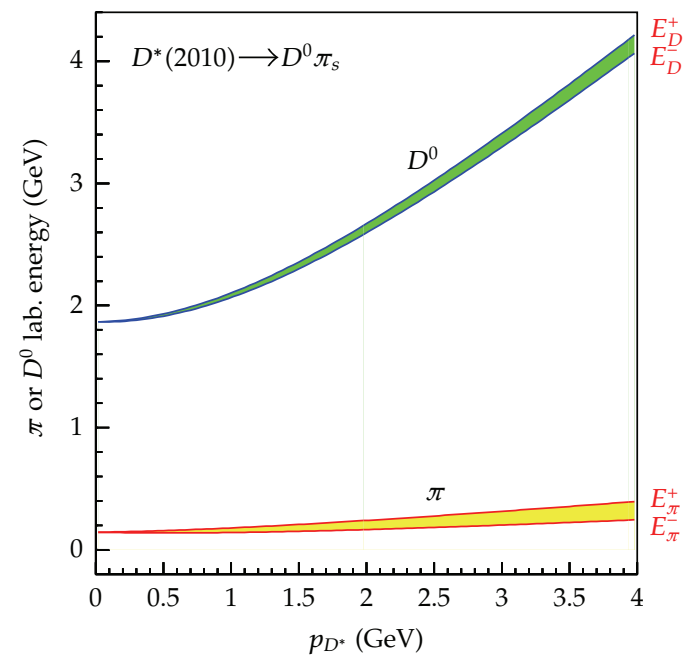

(c)

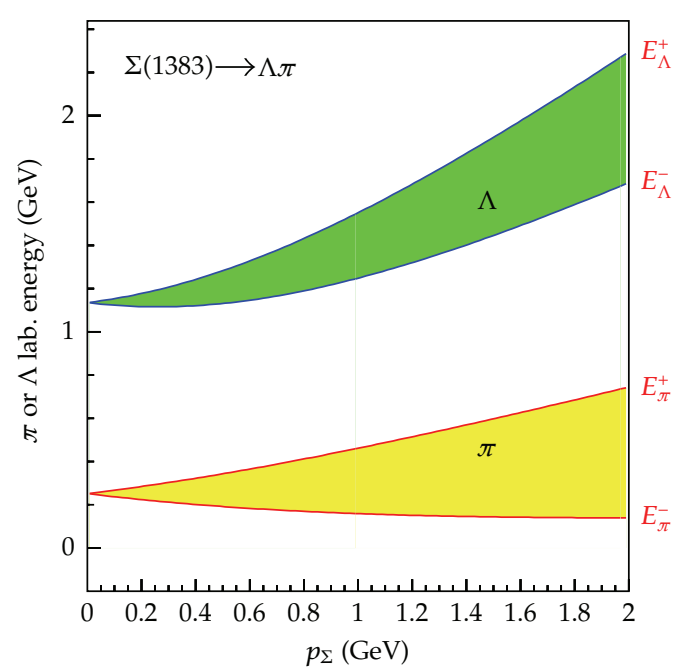

(b)

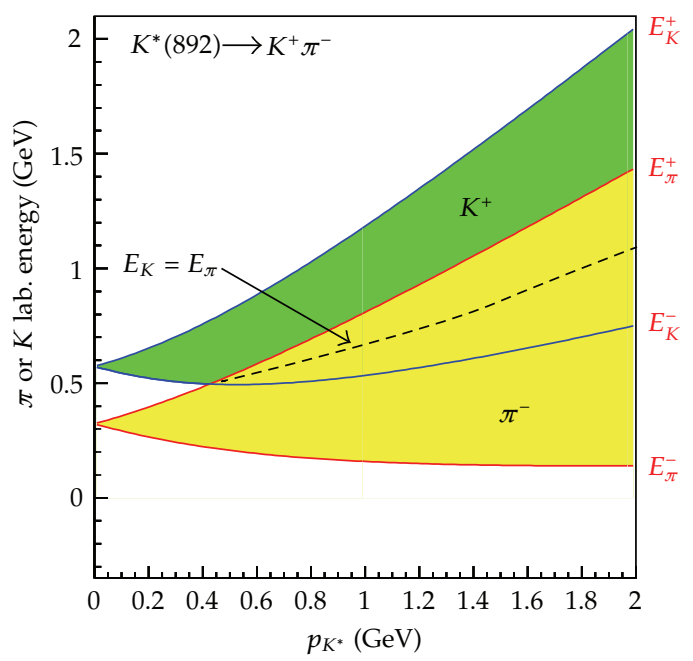

(d)

Figure 2: Phase space $E$-bands in $R \rightarrow \mathbf{a}+\mathbf{b}$ decays as a function of the resonance momentum $P_{R}$. The dashed line on (d) corresponds to (2.20).

In the case under consideration, $\cos \widehat{\theta}_{\mathbf{a}}^{*}$ is a solution of the equation:

$$
E_{\mathbf{a}}\left(\cos \theta_{\mathbf{a}}^{*}, P_{R}\right)=E_{\mathbf{b}}\left(\cos \theta_{\mathbf{b}}^{*}, P_{R}\right)
$$

Thus,

$$
\cos \widehat{\theta}_{\mathbf{a}}^{*}=-\frac{E_{R}}{P_{R}} \cdot \frac{\left(E_{\mathbf{a}}^{*}-E_{\mathbf{b}}^{*}\right)}{2 P^{*}}
$$

In the limit large $P_{R}$, with (2.21) we again recover (2.15)-(2.16). The evolution of Eff in $K^{*} \rightarrow K \pi$ decays is shown in Figure 4 (a) by the full line. Eff $=100 \%$ at $P_{R}<\widehat{P}_{R}$ and drops down up to the value (2.15), Eff $\simeq 71 \%$, with an increase in $P_{R}$. 


\section{Remark about $D^{*}$ Reconstruction}

Charged particles are tracked in a tracking detector (TD). The resolution of the transverse momentum of a track traversing the TD is parametrized by $\sigma\left(p_{T}\right) / p_{T}=A \cdot p_{T} \oplus B \oplus C / p_{T}$, with $p_{T}$ being the track transverse momentum (in $\mathrm{GeV}$ ). The coefficients $A, B$, and $C$ characterize the resolution of the TD. Usually, to increase the momentum resolution, only tracks with $p_{T}>0.12-0.15 \mathrm{GeV}$ are selected. That cut, as shown in Figure 1(c), makes impossible the reconstruction of $D^{*}$ mesons with momenta lower than $1.8-2.0 \mathrm{GeV}$. The $m$-band of $\pi$ mesons is very narrow and grows rather slowly with $P_{R}$. Tracks with momenta greater than $p_{\pi}^{+}\left(P_{D^{*}}\right)$ belong to the background. This property can be used to suppress the background in the distribution on the mass difference, $\Delta M=M\left(K \pi \pi_{s}\right)-M(K \pi)$, by imposing on the momentum of the soft pion $\pi_{s}$, the following cut,

$$
p_{\pi_{s}}<p_{0}+p_{\pi}^{+}\left(P_{D^{*}}\right)
$$

where $p_{0}=0.0-0.3 \mathrm{GeV}$ is a some shift from the pion $m$-band. In the decay $D^{*} \rightarrow D^{0} \pi$, the rest frame momentum is small, $P^{*}=0.038 \mathrm{GeV}$, and the $D^{*}$ momentum can be estimated with (2.6) by means of the reconstructed $D^{0}$ momentum:

$$
P_{D^{*}}=\frac{M_{D^{*}}}{M_{D^{0}}} \cdot P_{D^{0}}
$$

Thus, from (3.2) and (2.6) one get with (3.1):

$$
p_{\pi_{s}}<p_{0}+\frac{\left(m_{\pi}+P^{*}\right)}{M_{D^{0}}} \cdot P_{D^{0}}
$$

Instead of (3.3), it is possible to apply another, less-strict cut:

$$
p_{\pi_{s}}<p_{\pi}^{+}\left(P_{\max }\right) \simeq \frac{\left(m_{\pi}+P^{*}\right)}{M_{D^{*}}} \cdot P_{\max } .
$$

Here, $P_{\max }$ is the right-hand edge of the momentum range of the $D^{*}$ candidates, $P_{D^{*}}<P_{\max }$.

\section{Pentaquark States}

Now we apply the results of the previous sections to new resonances predicted $[8,9]$ by Diakonov, Petrov, and Polyakov in the framework of the chiral soliton model and detected both in the formation type [4,10-18] and in the production type [3] experiments. This is socalled five-quark states (pentaquarks). For a review on pentaquark phenomenology, see [19].

4.1. $\Theta(1530) \rightarrow N(939)+K(498)$

With the mass value predicted for $\theta^{+}, M_{\theta}=1.530 \mathrm{GeV}$, and the masses (in calculations were used mass values averaged over the isomultiplet) of decay products, $m_{N}=0.939 \mathrm{GeV}$ and $m_{K}=0.498 \mathrm{GeV}$, the condition (2.9) is not satisfied. That implies the overlap of the $m$-bands 
at all $P_{\theta}$ (Figure 3(a)) as well as the overlap of the E-bands at $P_{\theta}>\widehat{P}_{\theta}=2.15 \mathrm{GeV}$ (Figure $3(\mathrm{~b})$ ). The overlapping is not strong and both selectors (2.11) and (2.18) work with high efficiency (see Figure 4). The $m$ - and $E$-selectors [2] were successfully applied in searches for the $\theta^{+}$in the production-type $[3,20,21]$ and the formation-type $[4,17]$ experiments.

The situation with the $\theta^{+}$particle searches is rather complicated and confusing, especially when similar experiments have different conclusions on a signal existence. To be more specific, let us remind that two similar collider experiments at DESY (Hamburg) $[3,23]$ and two similar fixed-target experiments at IHEP (Protvino) [4, 24] do not agree on the existence of an exotic state with the mass close to $1530 \mathrm{MeV}$, which may be interpreted as a strange pentaquark state $\theta$ (1530) [8]. While the ZEUS [3] and SVD-2 [4] experiments claim the observation of a narrow peak near $1530 \mathrm{MeV}$ in the decay mode $\theta^{+} \rightarrow p K_{S}^{0}$, the $\mathrm{H} 1$ and SPHINX collaborations do not see it. The inconsistency of the experimental situation with searches of $\theta^{+}$baryon has risen even more, after the recent public polemic on results obtained with the CLAS detector at Thomas Jefferson National Accelerator Facility [25, 26]. All this discussion is beyond the scope of the present paper and can be found elsewhere $[27,28]$.

\section{2. $\Xi_{3 / 2}(2070) \rightarrow \Xi(1318)+\pi(139)$}

A baryonic state $\Xi$ is a weakly decaying particle. In a cascade decay, the vertex of the decay $\Xi \rightarrow \Lambda \pi$ is separated from the primary vertex, similarly to $\Lambda \rightarrow p \pi^{-}$decays. This property is used to suppress combinations which do not result from a $\Xi$ decay and helps to reconstruct the $\Xi$ candidate and its invariant mass.

In spite of the large mass asymmetry, $m_{\Xi} \gg m_{\pi}$, the conditions (2.9)-(2.10) are not fulfilled in $\Xi_{3 / 2}$ decays, and the pictures of the $m$ - and $E$-bands shown in Figures 3(c) and $3(\mathrm{~d})$ are very similar to those in $\Theta^{+}$decays. At low momenta, the efficiency of the $E$-selector is $100 \%$. The $E$-bands start overlapping at $P_{\Xi}>1.96 \mathrm{GeV}$. Thus, the efficiency of the $E$-selector is no worse than $84 \%$ (Figure 4 ).

4.3. $\Xi_{3 / 2}(2070) \rightarrow \Sigma(1385)+K(498)$

The $\Sigma(1385)$ decays strongly and exclusively at the primary event vertex. The decay modes $\Sigma^{ \pm 0} \rightarrow \Lambda \pi^{ \pm 0}$ are dominant. Therefore, after the reconstruction of $\Lambda$, the use of the $m$ - or $E$-selector allows reconstruction of the $\Sigma(1385)$ with high efficiency (see Figures $1(\mathrm{~b})$ and 2(b)).

Figures 3(e) and 3(f) show the $m$ - and $E$-bands in $\Xi_{3 / 2}(2070)$ decays. The $m$-bands slightly overlap at low $P_{\Xi}$ and diverge at $P_{\Xi}>7 \mathrm{GeV}$. The $E$-bands are totally separated. Thus, in that decay mode, the $m$ - and $E$-selectors work with $100 \%$ efficiency at high $P_{\Xi}$ momenta (Figure 4).

\section{4. $\Xi_{3 / 2}(2070)$ or $\Xi_{3 / 2}(1860)$ ?}

The NA49 collaboration provided an evidence for the existence in the decay mode $\Xi^{-} \pi^{-}$a narrow baryonic state with the mass of $1.862 \pm 0.002 \mathrm{GeV}$ [22]. This state is considered as a candidate for the exotic pentaquark state $\Xi_{3 / 2}$. The reported mass value is much lower as predicted in [8]. The last developments in the theory of pentaquark states did not exclude the lower mass for the $\Xi_{3 / 2}$ [9]. In Figure 5 are shown the $m$-bands and the efficiency of both 


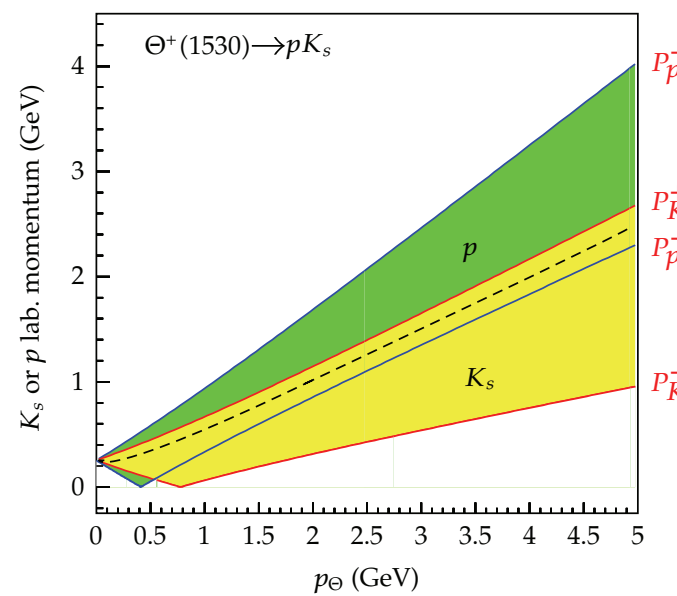

(a)

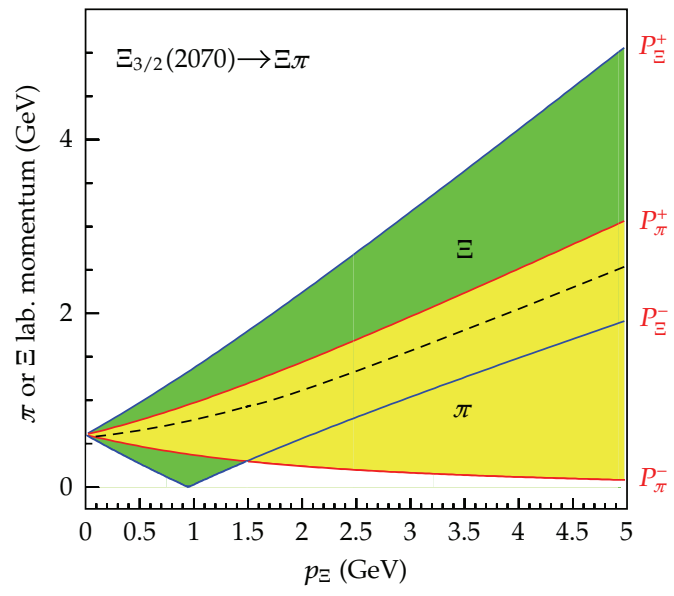

(c)

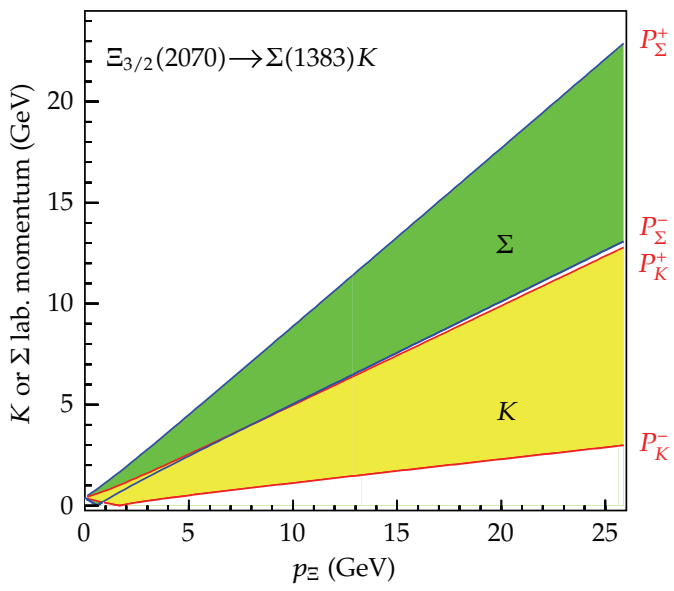

(e)

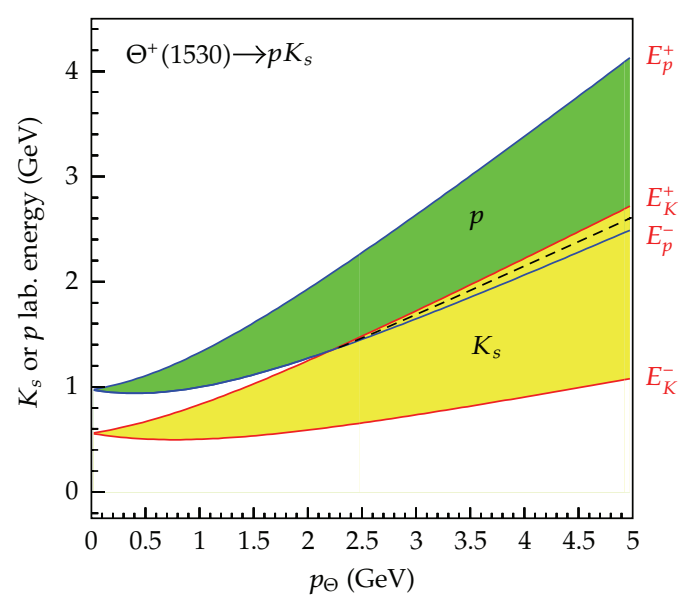

(b)

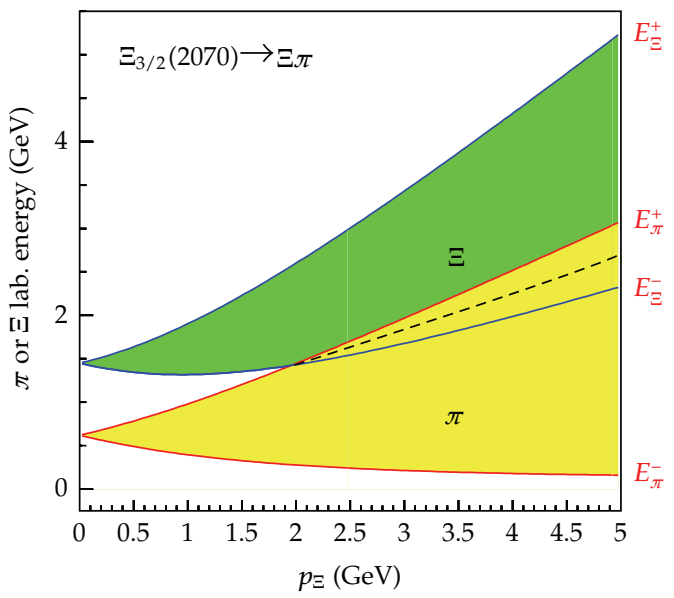

(d)

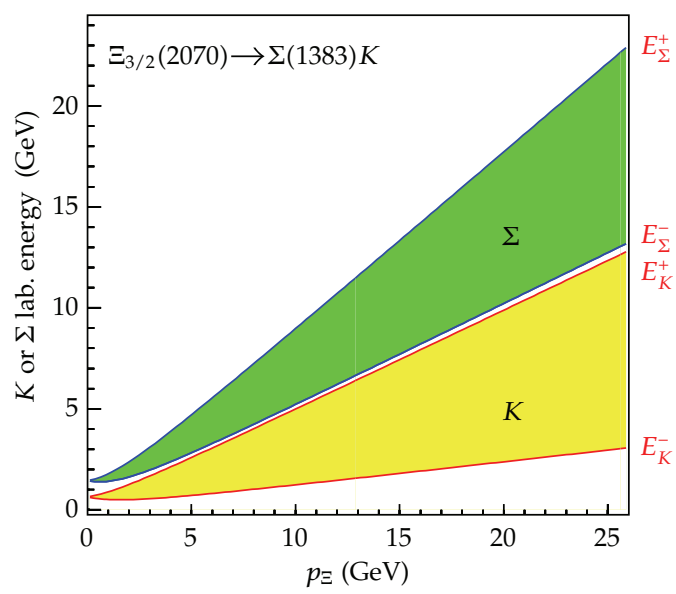

(f)

Figure 3: Phase space $m$-bands ((a), (c), and (e)) and $E$-bands ((b), (d), and (f)) in decays of the pentaquark states $\Theta(1530)$ and $\Xi_{3 / 2}(2070)$. The dashed lines correspond to (2.12) and (2.20). 


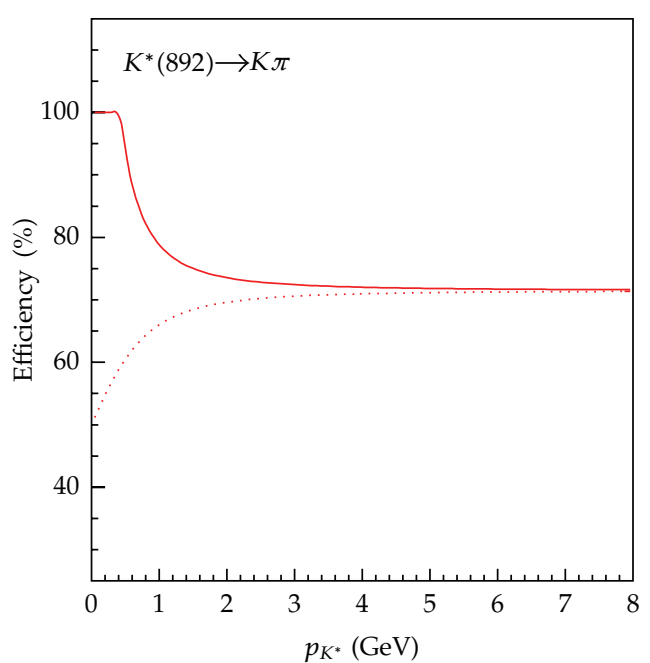

(a)

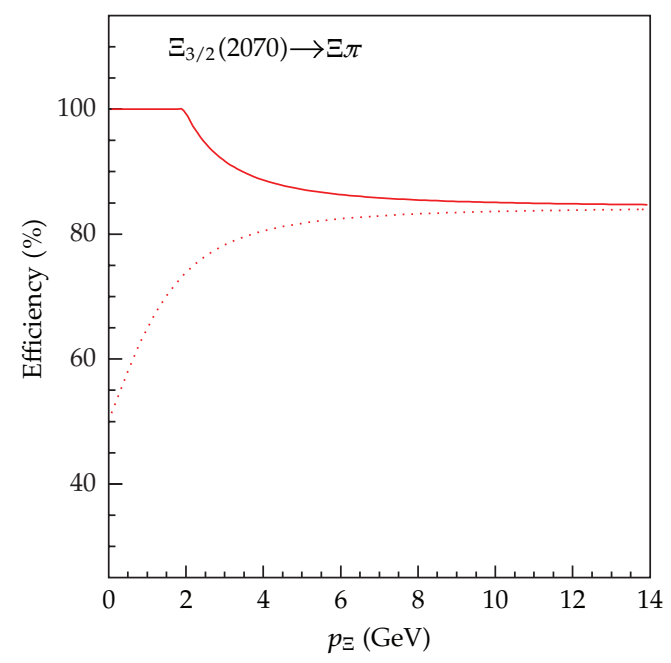

(c)

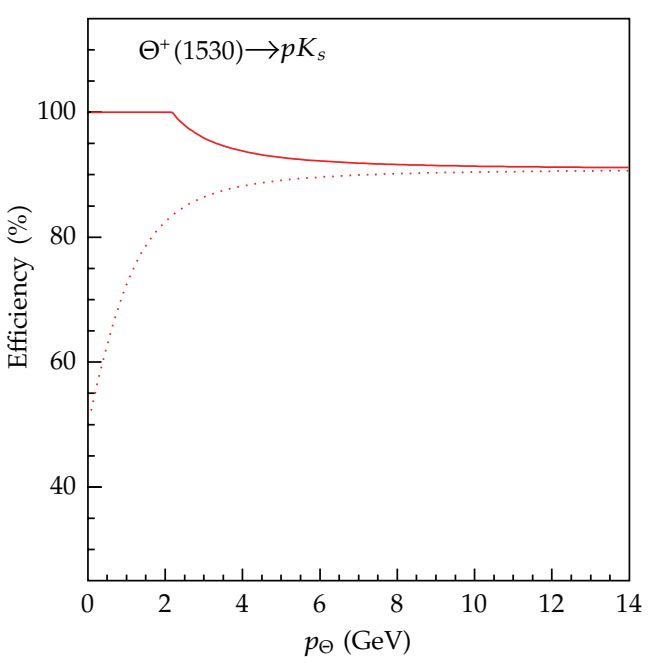

(b)

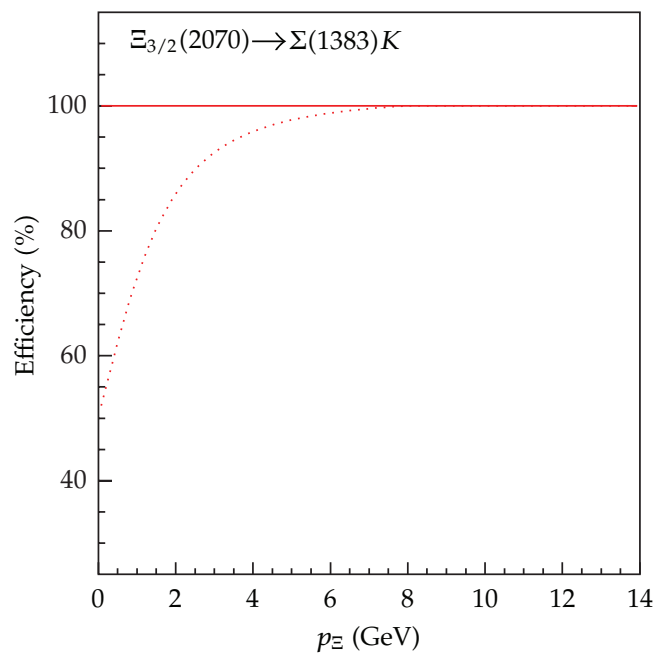

(d)

Figure 4: Efficiency of the selection rule $p_{\mathbf{a}}>p_{\mathbf{b}}$ (dashed line) and $E_{\mathbf{a}}>E_{\mathbf{b}}$ (full line) at different $P_{R}$ in decays of $K^{*}(892), \Theta(1530)$, and $\Xi_{3 / 2}(2070)$ resonances.

selectors. They are much the similar to those in Figures 3(e) and 4(d). The picture of the $E$ bands is also similar to Figure 3(f). Thus, the E-selector will not suppress the signal but will suppress the background at higher masses.

We have to note that there are arguments [29] that the result of the NA49 collaboration perhaps is inconsistent with data collected over the past decades.

\section{Conclusions}

Kinematics of the two-body decay, $R \rightarrow \mathbf{a}+\mathbf{b}$, has been analyzed in terms of equations describing boundaries of the physical region in decays and the graphical phase space $m$ - and $E$-bands. The efficiency of the $m$ - and $E$-selectors was estimated with the use of (2.14). On 


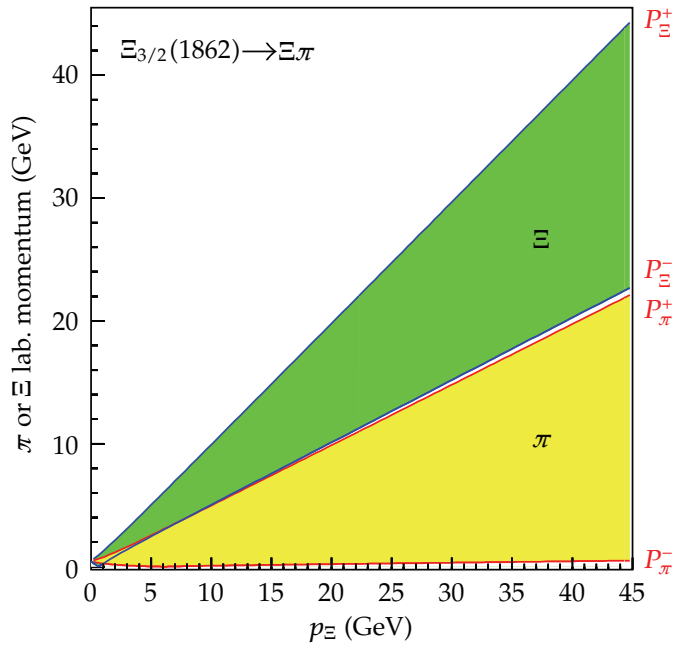

(a)

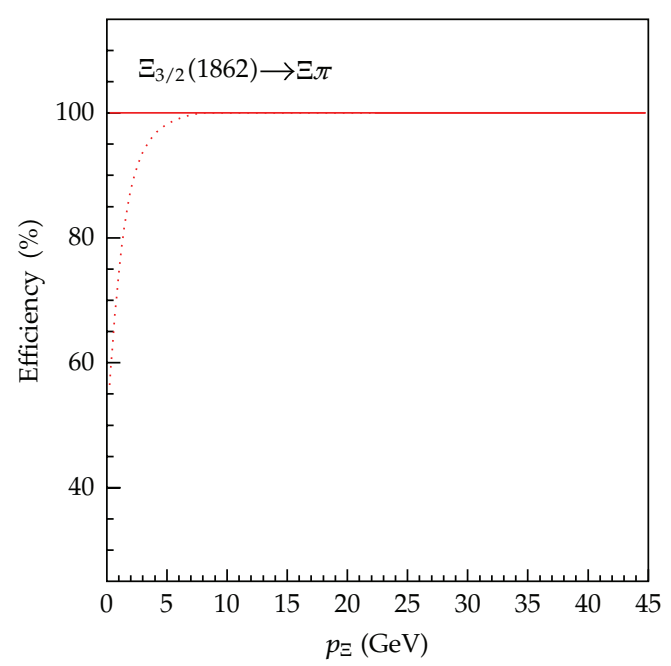

(b)

Figure 5: The $m$-bands and the efficiency of the $m$ - and $E$-selectors for the $\Xi_{3 / 2} \rightarrow \Xi+\pi$ decay with the $\Xi_{3 / 2}$ mass reported by the NA49 collaboration [22].

the basis of many examples, in particular, the exotic antidecuplet baryons (pentaquark states), it has been demonstrated that for $m_{\mathrm{a}}>m_{\mathrm{b}}$ the selection rules $p_{\mathrm{a}}>p_{\mathbf{b}}$ and $E_{\mathbf{a}}>E_{\mathbf{b}}$ can be with a high efficiency applied for the signal reconstruction from many resonances.

\section{Dedication}

This work is dedicated to the memory of Professor Pavel F. Ermolov (30.06.1932-14.05.2008), a prominent physicist, the organizer of Department of Experimental High Energy Physics, SINP MSU, the main "driving force" of the SVD experiment and a wonderful person.

\section{Acknowledgments}

The author is indebted to P. Bussey and S. Chekanov for comments and grateful to other colleagues from the ZEUS collaboration for useful discussions. Special thanks are due to P. F. Ermolov and A. Kubarovsky for discussions of preliminary results of the SVD collaboration.

\section{References}

[1] ZEUS Collaboration, S. Chekanov et al., "Observation of $K_{S}^{0} K_{S}^{0}$ resonances in deep inelastic scattering at HERA," Physics Letters B, vol. 578, p. 33, 2004.

[2] B. B. Levchenko, "Kinematic selection criteria in a new resonance searches: application to pentaquark states," http://arxiv.org/abs/hep-ph/0401122.

[3] ZEUS Collaboration, S. Chekanov et al., "Evidence for a narrow Baryonic state decaying to $K_{s}^{0}$ (anti)proton in deep inelastic scattering at HERA," Physics Letters B, vol. 591, p. 7, 2004.

[4] SVD Collaboration, A. Aleev et al., "Observation of a narrow Baryon resonance decaying into $p K_{s}^{0}$ in pA-interactions at $70 \mathrm{GeV} / \mathrm{c}$ with SVD-2 setup," Physics of Atomic Nuclei, vol. 68, p. 974, 2005.

[5] G. I. Kopilov, Basics of Resonance Kinematics, Nauka, 1970. 
[6] E. Byckling and K. Kajantie, Particle Kinematics, John Wiley \& Sons, 1972.

[7] K. Nakamura, (Particle Data Group) et al., "Review of particle physics," Journal of Physics G, vol. 37, Article ID 075021, 2010.

[8] D. Diakonov, V. Petrov, and M. V. Polyakov, "Exotic anti-decuplet of Baryons: prediction from chiral solitons," Zeitschrift für Physik A, vol. 359, p. 305, 1997.

[9] M. Polyakov, "DESY Forum: pentaquarks at HERA, DESY, Hamburg," 2003, http:/ / webcast.desy.de.

[10] LEPS Collaboration, T. Nakano et al., "Evidence for a narrow $S=+1$ Baryon resonance in photoproduction from the neutron," Physical Review Letters, vol. 91, Article ID 012002, 2003.

[11] CLAS Collaboration, S. Stepanyan et al., "Observation of an exotic $S=+1$ Baryon in exclusive photoproduction from the Deuteron," Physical Review Letters, vol. 91, Article ID 252001, 2003.

[12] CLAS Collaboration, V. Kubarovsky et al., "Observation of an exotic Baryon with $S=+1$ in photoproduction from the proton," Physical Review Letters, vol. 92, Article ID 032001, 2004, Erratumibid. vol.92, Article ID, 049902, 2004.

[13] SAPHIR Collaboration, J. Barth, J. Ernst et al., "Evidence for the positive-strangeness pentaquark $\theta^{+}$in photoproduction with the SAPHIR detector at ELSA," Physics Letters B, vol. 572, no. 3-4, pp. 127-132, 2003.

[14] A. E. Asratyan, A. G. Dolgolenko, and M. A. Kubantsev, "Evidence for formation of a narrow $p K_{s}$ resonance with mass near $1533 \mathrm{MeV}$ in neutrino interactions," Physics of Atomic Nuclei, vol. 67, no. 4, pp. 682-687, 2004.

[15] DIANA Collaboration, V. V. Barmin, V. S. Borisov et al., "Observation of a Baryon resonance with positive strangeness in $K^{+}$collisions with Xe nuclei," Physics of Atomic Nuclei, vol. 66, no. 9, pp. 17151718, 2003.

[16] HERMES Collaboration, A. Airapetian et al., "Evidence for a narrow $|S|=1$ Baryon state at a mass of $1528 \mathrm{MeV}$ in quasi-real photoproduction," Physics Letters B, vol. 585, p. 213, 2004.

[17] SVD Collaboration, A. Aleev et al., "Further study of the narrow Baryon resonance decaying into $K_{s}^{0} p$ in pA-interactions at $70 \mathrm{GeV} / \mathrm{c}$ with SVD-2 setup," arXiv:hep-ex/0509033v3.

[18] SVD Collaboration, A. Aleev et al., "New analysis on the narrow Baryon resonance decaying into $p K_{s}^{0}$ in pA-interactions at $70 \mathrm{GeV} / \mathrm{c}$ with SVD-2 setup ," arXiv:0803.3313v2.

[19] R. L. Jaffe, "Exotica," Physics Reports, vol. 409, no. 1, pp. 1-45, 2005.

[20] S. V. Chekanov and B. B. Levchenko, "The first observation of the $1530 \mathrm{MeV}$ peak in $K_{s}^{0} p$ mode with the ZEUS HERA I data," A presentation at the QCD working group, ZEUS.

[21] ZEUS Collaboration and S. Chekanov, "DESY Forum: Pentaquarks at HERA, DESY, Hamburg," 2003, http:/ / webcast.desy.de./

[22] NA49 Collaboration, C. Alt et al., "Observation of an exotic $S=-2, Q=-2$ Baryon resonance in proton-proton collisions at the CERN SPS," Physical Review Letters, vol. 92, Article ID 042003, 2004.

[23] H1 Collaboration, A. Aktas et al., "Search for a narrow Baryonic resonance decaying to $K_{s}^{0} p$ or $K_{s}^{0} \bar{p}$ in deep inelastic scattering at HERA," Physics Letters B, vol. 639, p. 202, 2006.

[24] SPHINX Collaboration, Y. M. Antipov et al., "Search for $\Theta^{+}(1540)$ in exclusive proton-induced reaction $\mathrm{p}+\mathrm{C}(\mathrm{N}) \rightarrow \Theta^{+} \bar{K}^{0}+\mathrm{C}(\mathrm{N})$ at the energy of $70 \mathrm{GeV}$, " European Physical Journal A, vol. 21, Article ID 040702, p. 455, 2004.

[25] M. J. Amaryan et al., "Observation of a narrow structure in ${ }^{1} \mathrm{H}\left(\gamma, K_{S}^{0}\right) X$ via interference with $\phi$-Meson production," Physical Review C, vol. 85, Article ID 035209, 2012.

[26] CLAS Collaboration, M. Anghinolfi et al., "Comment on the narrow structure reported by Amaryan et al," Physical Review C, http:/ / arxiv.org/abs/1204.1105v1.

[27] S. V. Chekanov and B. B. Levchenko, "Influence of experimental resolution on the statistical significance of a signal: implication for pentaquark searches," Physical Review D, vol. 76, no. 7, Article ID 074025, 2007.

[28] Y. Azimov, K. Goeke, and I. Strakovsky, "Explanation why the $\Theta^{+}$is seen in some experiments and not in others," Physical Review D, vol. 76, no. 7, Article ID 074013, 2007.

[29] H. G. Fischer and S. Wenig, "Are there S = -2 pentaquarks?" European Physical Journal C, vol. 37, no. 2, pp. 133-140, 2004. 

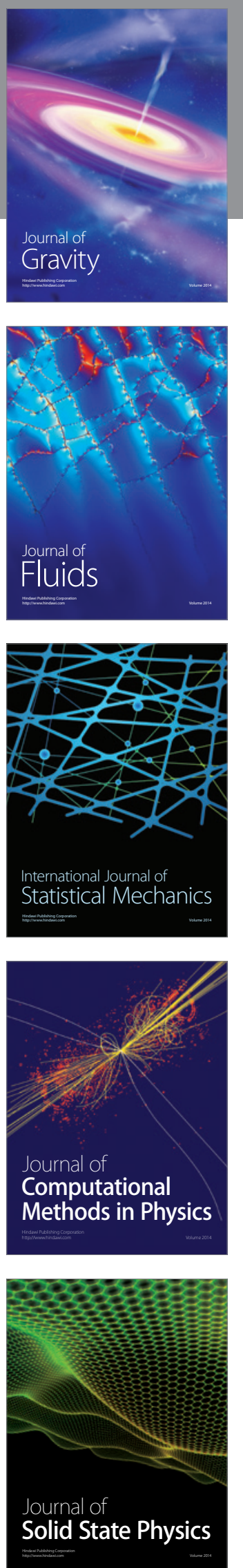

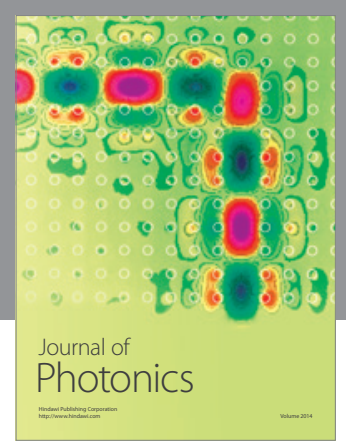

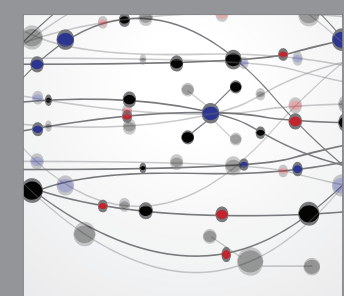

The Scientific World Journal
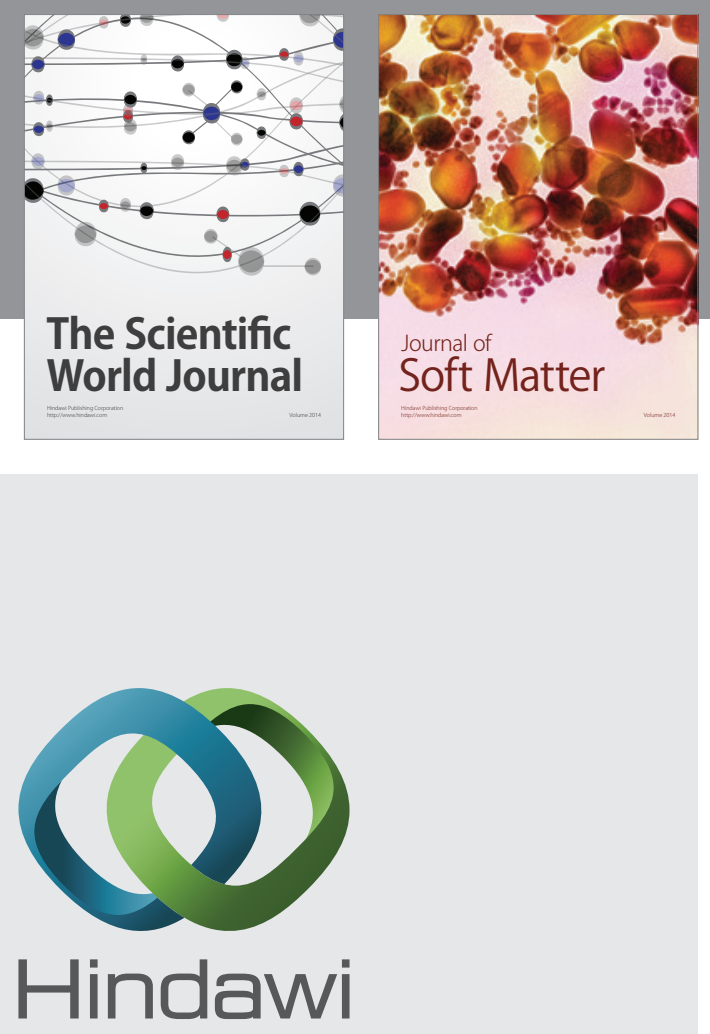

Submit your manuscripts at

http://www.hindawi.com
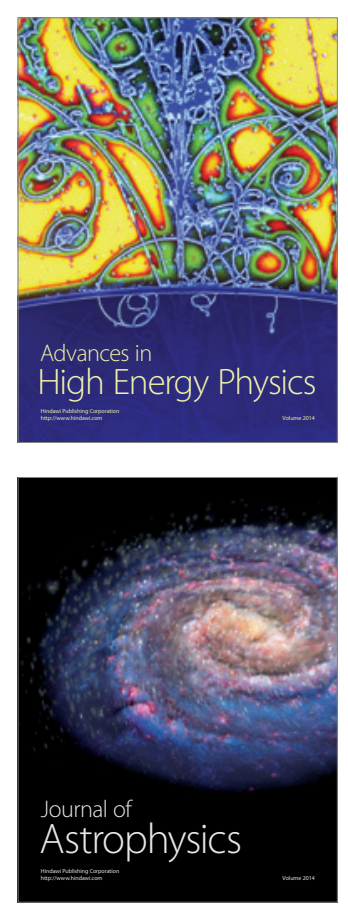
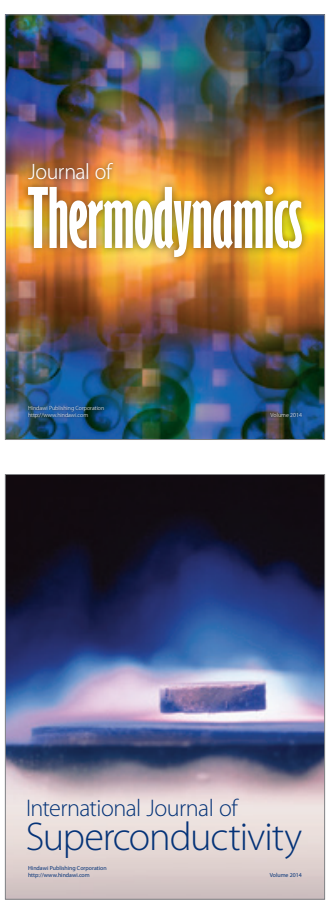
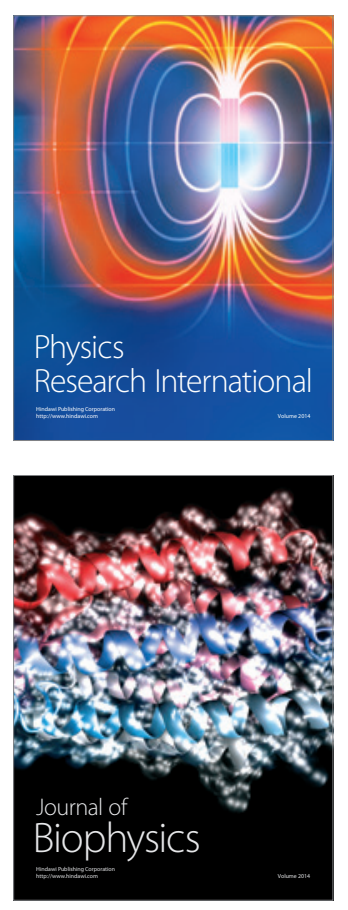
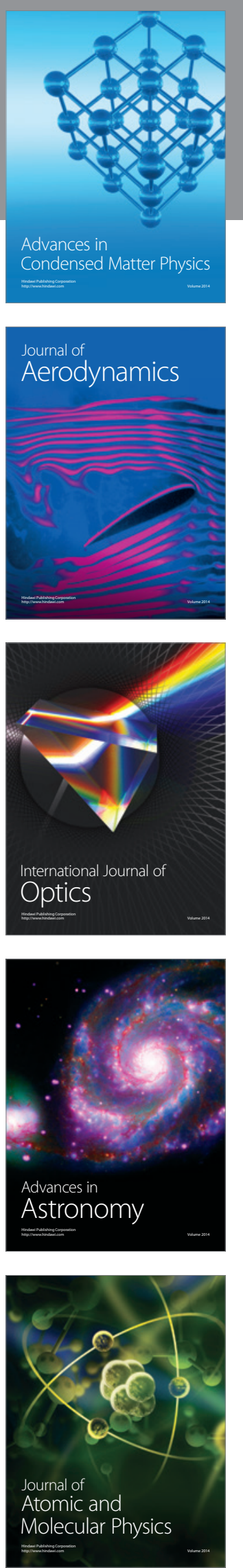